\title{
Accurate calculation of the bound states of Hellmann potential
}

\author{
Amlan K. Roy* \\ Department of Chemistry and Biochemistry, \\ University of California, Los Angeles, CA, 90095-1569, USA \\ Abraham F. Jalbout \\ Institute of Chemistry, National Autonomous University of Mexico, Mexico City, Mexico \\ Emil I. Proynov \\ Q-Chem Inc., 5001 Baum Blvd., Pittsburgh, PA 15213, USA
}

\begin{abstract}
Bound states of the Hellmann potential, which is a superposition of the attractive Coulomb $(-A / r)$ and the Yukawa $\left(B e^{-C r} / r\right)$ potential, are calculated by using a generalized pseudospectral method. Energy eigenvalues accurate up to thirteen to fourteen significant figures, and densities are obtained through a nonuniform, optimal spatial discretization of the radial Schrödinger equation. Both ground and excited states are reported for arbitrary values of the potential parameters covering a wide range of interaction. Calculations have been made for higher states as well as for stronger couplings. Some new states are reported here for the first time, which could be useful for future works. The present results are significantly improved in accuracy over all other existing literature values and offers a simple, accurate and efficient scheme for these and other singular potentials in quantum mechanics.
\end{abstract}

\footnotetext{
${ }^{*}$ Corresponding author. Email: akroy@chem.ucla.edu
} 


\section{INTRODUCTION}

A two-particle system interacting through a combination of the attractive Coulomb and the Yukawa potential,

$$
v(r)=-A / r+B e^{-C r} / r
$$

has received considerable interest for several decades. In this equation, the parameters $A$, $B$ characterize the strength of the Coulomb and Yukawa potentials respectively; $C$ is the screening parameter and $r$ signifies the distance between the two particles. $A, C$ are positive and $B$ can be both positive as well as negative. Historically, such a superposed potential with positive $B$ was first studied by Hellmann [1-3] long times ago and thereafter has been customarily used to include both positive and negative $B$. This has found various important applications in the field of atomic and condensed matter physics; e.g., the electron-core $[4,5]$, electron-ion [6,7], inner-shell ionization [8] problems, alkali hydride molecules [9], solid-state physics $[10,11]$, etc.

From the theoretical perspectives, this potential has attracted considerable attention from various workers. Like most other practical physical systems, the corresponding Schrödinger equation (SE) does not offer exact analytical solutions in this case too, and one has to resort to the approximate methodologies, such as the variational or the perturbative approaches. Some important aspects of the bound-state spectra of this system are the presence of complex level crossings [12] and the absence of accidental degeneracies (characteristics of the pure Coulomb potential). Quite detailed calculations were performed [12] using the variational technique including ten parameters for wide ranges of the parameters in the potential corresponding to ground, as well as low and moderately high values of the $n$ and $\ell$ quantum numbers. Shortly after that, shifted large N expansion results [13] were reported for these systems which were more or less of similar accuracy as those of [12], although with limited applicability. Besides, attempts have been made to use first-order Rayleigh-Schrödinger perturbation theory to provide approximate analytical formulas for the bound eigenstates [14]. Lately, a combined Hellmann-Feynmann theorem and the principle of minimal sensitivity has also been used to investigate these states [15]. Analytical formulas for the upper and lower bounds [16] have been presented recently by using an envelope method in conjunction with the comparison theorem. However, despite all these elegant formalisms, there are several problems which deserve more careful and thorough examinations. For example, the 
prescription of [13] yields reasonably good results for very weak screenings and gradually worsens as $B$ and $C$ increase. For certain other choices of the parameters, this leads to divergent energy series for some of the eigenstates. Analogous difficulties have also been faced in the recent treatment of these potentials [15] using the shifted $1 / \mathrm{N}$ expansion. It is also worthwhile to note that although the variational results of [12] were quite accurate and so far have been used as a standard in the literature for this potential, it would be useful and desirable to have more accurate results for these states. The lack of such results in the literature is little surprising, especially in the light of the fact that many excellent and high quality results have been available for both the Coulomb and Yukawa potentials for many years (see for example [17-19]). Thus a general reliable formalism which can offer accurate and physically meaningful results for arbitrary values of the interaction parameters for both low as well as higher states, would have its own merit.

Hence it would be of some interest to investigate the spectra of these systems with a fresh look. The purpose of this Letter is to employ the generalized pseudospectral (GPS) method to solve the corresponding SE in a simple and accurate manner. To this end, accurate eigenvalues and densities are reported for all the $n \leq 5$ states and the effects of varying the interaction parameters are studied by covering a large range. The GPS method has emerged as a quite successful formalism to study a multitude of atomic and molecular processes in the past years including both electronic structure and dynamics calculations having Coulomb singularities. Recently it has also been shown to be equally successful for the spiked harmonic oscillators, logarithmic and power-law potentials, the Hulthen and Yukawa potentials as well as other singular potentials [20-23]. Comparisons with the literature data have been made wherever possible. The article is organized as follows: Section II gives a brief outline of the GPS method used here to solve the SE in presence of the Hellmann potential. A discussion of the results is made in Section III, while we end with a few concluding remarks in Section IV.

\section{THE GPS FORMALISM FOR THE SOLUTION OF HELLMANN POTENTIAL}

This section presents an overview of the GPS formalism along with the mapping procedure used for solving the radial SE of a Hamiltonian containing a Hellmann potential within the nonrelativistic framework. Only the essential steps are given and the relevant details may 
be found elsewhere ([20-23] and the references therein). Unless otherwise mentioned, atomic units are employed throughout this article.

The radial SE can be written in the following form,

$$
\left[-\frac{1}{2} \frac{\mathrm{d}^{2}}{\mathrm{~d} r^{2}}+\frac{\ell(\ell+1)}{2 r^{2}}+v(r)\right] \psi_{n, \ell}(r)=E_{n, \ell} \psi_{n, \ell}(r)
$$

where $v(r)$ is given as in Eq. (1). Here $n$ and $\ell$ signify the usual radial and angular momentum quantum numbers respectively.

One of the distinctive features of GPS method is that it allows one to work in a nonuniform and optimal spatial discretization; a coarser mesh at larger $r$ and a denser mesh at smaller $r$, while maintaining a similar accuracy at both the regions. Thus it suffices to work with a significantly smaller number of grid points efficiently, which is in sharp contrast to some of the commonly used finite difference or finite element methods for the singular potentials, where one is almost forced to use considerably larger mesh, often presumably because of their uniform nature.

At the first step a function $f(x)$ defined in the interval $x \in[-1,1]$ is approximated by the N-th order polynomial $f_{N}(x)$ as follows,

$$
f(x) \cong f_{N}(x)=\sum_{j=0}^{N} f\left(x_{j}\right) g_{j}(x),
$$

which guarantees that the approximation is exact at the collocation points $x_{j}$, i.e.,

$$
f_{N}\left(x_{j}\right)=f\left(x_{j}\right)
$$

In the Legendre pseudospectral method which we use here, $x_{0}=-1, x_{N}=1$, and the $x_{j}(j=1, \ldots, N-1)$ are obtained from the roots of the first derivative of the Legendre polynomial $P_{N}(x)$ with respect to $x$, i.e.,

$$
P_{N}^{\prime}\left(x_{j}\right)=0
$$

The $g_{j}(x)$ in Eq. (3) are called the cardinal functions given by,

$$
g_{j}(x)=-\frac{1}{N(N+1) P_{N}\left(x_{j}\right)} \frac{\left(1-x^{2}\right) P_{N}^{\prime}(x)}{x-x_{j}},
$$

and satisfy the unique property, $g_{j}\left(x_{j^{\prime}}\right)=\delta_{j^{\prime} j}$. At this stage one can map the semi-infinite domain $r \in[0, \infty]$ onto the finite domain $x \in[-1,1]$ by the transformation $r=r(x)$. Now introduction of the following algebraic nonlinear mapping,

$$
r=r(x)=L \frac{1+x}{1-x+\alpha},
$$


where $\mathrm{L}$ and $\alpha=2 L / r_{\max }$ are the mapping parameters, in conjunction with the relation,

$$
\psi(r(x))=\sqrt{r^{\prime}(x)} f(x)
$$

followed by a symmetrization procedure leads to the transformed Hamiltonian as below,

$$
\hat{H}(x)=-\frac{1}{2} \frac{1}{r^{\prime}(x)} \frac{d^{2}}{d x^{2}} \frac{1}{r^{\prime}(x)}+v(r(x))+v_{m}(x),
$$

where $v_{m}(x)$ is given by,

$$
v_{m}(x)=\frac{3\left(r^{\prime \prime}\right)^{2}-2 r^{\prime \prime \prime} r^{\prime}}{8\left(r^{\prime}\right)^{4}} .
$$

This has the advantage that one deals with a symmetric matrix eigenvalue problem which can be easily solved by standard available routines to yield accurate eigenvalues and eigenfunctions. Note that $v_{m}(x)=0$ for the particular transformation.

We have carried out a large number of tests in order to make a detailed check on the accuracy and reliability of the method by varying the mapping parameters so as to produce "stable" results with respect to their changes. This procedure was applied for a variety of potential parameters available in the literature. In this way, a consistent set of parameters $\alpha=25, N=200$ and $r_{\max }=200$ were chosen which seemed to be appropriate for all the calculations performed in this work. The results are reported only up to the precision that maintained stability and all our results are truncated rather than rounded-off. Thus, all the results may be considered as correct up to the place they are reported.

\section{RESULTS AND DISCUSSION}

First in table 1 , we give the computed $2 s$ eigenvalues for three values of the parameter $B$, viz., $0.5,-0.5$, and -2 as a function of the screening parameter $C$ to demonstrate the accuracy of the present calculations. For each $B$, four $C$ values have been considered covering both the weak and strong regions. It may be noted here that for all the calculations in this work, we assume $A=1$; accordingly our $B$ maps to half of the corresponding rescaled parameter of [12], and the computed eigenvalues obtained in a. u., are half of those of [12]. The results are compared with the (a) variational results [12], and (b) Rayleigh-Schrödinger perturbation calculations [14]. For $C=0.001$, only the former results are available. It is abundantly clear that the present GPS values are significantly better than either of these

previously reported results in all the cases. For all these states, between [12] and [14], 
TABLE I: Comparison of the calculated negative eigenvalues (in a.u.) with the literature for the $2 s$ states as functions of $B$ and $C$. Literature results have been appropriately converted to the current scale of units.

\begin{tabular}{cccccccc}
\hline \hline$B$ & $C$ & $-\mathrm{E}$ (This work) & $-\mathrm{E}($ Literature $)$ & $B$ & $C$ & $-\mathrm{E}$ (This work) & $-\mathrm{E}($ Literature $)$ \\
\hline 0.5 & 0.001 & 0.03174701400990 & $0.031745^{a}$ & 0.5 & 0.005 & 0.03367675354994 & $0.033675^{a, b}$ \\
0.5 & 2 & 0.11290716132278 & $0.112905^{a}, 0.11115^{b}$ & 0.5 & 10 & 0.12339007950313 & $0.12289^{a}, 0.123285^{b}$ \\
-0.5 & 0.001 & 0.2807509984473 & $0.28075^{a}$ & -0.5 & 0.005 & 0.2787748073142 & $0.278775^{a}, 0.27877^{b}$ \\
-0.5 & 2 & 0.1406129511670 & $0.14061^{a}, 0.13943^{b}$ & -0.5 & 10 & 0.1268366598878 & $0.126835^{a}, 0.126715^{b}$ \\
-2 & 0.001 & 1.1230019984462 & $1.12300^{a}$ & -2 & 0.005 & 1.1150498066913 & $1.115050^{a}, 1.115035^{b}$ \\
-2 & 2 & 0.2010044938456 & $0.201005^{a}, 0.20219^{b}$ & -2 & 10 & 0.1342619146710 & $0.13424^{a}, 0.13187^{b}$ \\
\hline \hline
\end{tabular}

${ }^{a}$ Ref. [12].

${ }^{b}$ Ref. [14].

former results are seen to be closer to present values than the latter ones. Usually the variationally calculated eigenvalues match up to four to five significant figures with those of ours while the perturbation results place these states at higher values in all but one instance $(B=-2, C=2)$ and the accuracy gradually decreases with an increase in the screening parameter $C$.

Next, the calculated binding energies $\left(-E_{n, \ell}\right)$ of some of the lowest lying $1 s-5 g$ states below $n \leq 5$ are presented for the strongly repulsive $(B=+5)$ and strongly attractive $(B=-5)$ Yukawa potentials in tables 2 and 3 respectively as a function of the screening parameter $C$. Four values of $C$ have been considered in both cases, viz., 0.01, 1, 10 and 100 which essentially covers both the weak and strong regions. $1 s-4 f$ states were studied for a large number of $C$ values ranging from $0.001-10$ by [12] while from $0.05-10$ by [13] and these are appropriately quoted here. To our knowledge, no reference values are available for $C>10$, and we report here some results in the very high screening regions $(C=100)$. It is noticed that, the present GPS eigenvalues are much superior to both the earlier reported values for all these states. For the repulsive Yukawa potential case, the variational results [12] are seen to match with our results in the moderate screening regions $(C=1,10)$; whereas, the accuracy in their result deteriorates for smaller $C(0.01)$. Also the low- $\ell$ states deviate more than the high- $\ell$ states. Furthermore, within a particular $\ell$, the errors increase as the radial quantum number $n$ increases. Thus the $1 s, 2 s, 3 s$ and $4 s$ states for $C=0.01$ are in error by $0.54,3.76,11.25$ and $23.25 \%$ respectively. However, for the attractive Yukawa potentials, they show similar kind of accuracies and agreements with the present results for 
TABLE II: Comparison of the calculated negative eigenvalues (in a.u.) with the literature for $B=+5$ as functions of the screening parameter $C$. Literature results have been appropriately converted to the current scale of units.

\begin{tabular}{cllll}
\hline \hline State & $C=0.01$ & $C=1$ & $C=10$ & $C=100$ \\
\hline $1 s$ & $0.002362763418\left(0.00235^{a}\right)$ & $0.1393937847772\left(0.139395^{a}\right)$ & $0.4219751601088\left(0.421975^{a}, 0.29851^{b}\right.$ & 0.4981833709122 \\
$5 s$ & 0.001525033897 & 0.0144970380925 & 0.0193116714697 & 0.0199854358123 \\
$5 d$ & 0.001862762081 & 0.0195295800293 & 0.0199999884584 & 0.0199999999999 \\
$4 f$ & $0.002300761996\left(0.00229^{a}\right)$ & $0.0312056245649\left(0.031205^{a}\right)$ & $0.0312499999917\left(0.03125^{a}, 0.03125^{b}\right)$ & 0.0312500000000 \\
$5 f$ & 0.002054101204 & 0.0199657840037 & 0.0199999999929 & 0.0200000000000 \\
$5 g$ & 0.002260639328 & 0.0199992848683 & 0.0199999999999 & 0.0199999999999 \\
\hline \hline
\end{tabular}

${ }^{a}$ Ref. [12].

${ }^{b}$ Ref. [13].

TABLE III: Comparison of the calculated negative eigenvalues (in a.u.) with the literature for $B=-5$ as functions of the screening parameter $C$. Literature results have been appropriately converted to the current scale of units.

\begin{tabular}{cllll}
\hline \hline State & $C=0.01$ & $C=1$ & $C=10$ & $C=100$ \\
\hline $1 s$ & $17.95006243069\left(17.95005^{a}\right)$ & 13.56679686030 & $0.9788396316974\left(0.97885^{a}\right)$ & 0.5020427358386 \\
$5 s$ & 0.6715273295205 & 0.0362575479838 & 0.0223084737740 & 0.0200162962261 \\
$5 d$ & 0.6714073958450 & 0.0214355247975 & 0.0200000117313 & 0.0200000000000 \\
$4 f$ & $1.075741875123\left(1.07575^{a}\right)$ & 0.0313020585157 & $0.0312500000082\left(0.03125^{a}, 0.0312^{b}\right)$ & 0.0312499999999 \\
$5 f$ & 0.6712874380109 & 0.0200407809113 & 0.0200000000070 & 0.0200000000000 \\
$5 g$ & 0.6711274566209 & 0.0200007358716 & 0.0200000000000 & 0.0200000000000 \\
\hline \hline
\end{tabular}

${ }^{a}$ Ref. [12].

${ }^{b}$ Ref. [13].

both $C=0.01$ and 10 . Once again, the variational results [12] are seen to be somewhat better than the perturbation results [13]. For the $1 s$ and $2 s$ states of $B=+5$ and $B=-5$, appreciable deviations are noticed in the calculations of [13] from ours $(\approx 29.26$ and $25.06 \%$ respectively); other $s$ states also suffer more compared to the $p, d$ or $f$ states. In both these cases, however, one regains the hydrogen-like spectrum in the limit of $C \rightarrow \infty$ (100 in the tables), as expected.

Now we examine the effects of the variation of parameter $B$ on the calculated eigenvalues up to $1 s$ through $5 g$. Tables 4 and 5 present such eigenvalues for moderate screenings $(C=0.25)$ at four $B$ values $(-10,-1,1,100)$ and strong screening $(C=1)$ at four $B$ values $(-25,-10,1,10)$ respectively. The reference values for these states are quite scarce. $n \leq 4$ 
TABLE IV: Comparison of the calculated negative eigenvalues (in a.u.) with the literature for $C=0.25$ as functions of $B$. Literature results have been appropriately converted to the current scale of units.

\begin{tabular}{cllll}
\hline \hline State & $B=-10$ & $B=-1$ & $B=1$ & $B=100$ \\
\hline $1 s$ & $58.04198638290\left(58.042^{a}\right)$ & $1.771691001196\left(1.77169^{a}\right)$ & $0.1105241235947\left(0.110525^{a}\right)$ & 0.0251808092657 \\
$5 s$ & 0.7519807159635 & 0.0271230027804 & 0.0142921176781 & 0.0067962156719 \\
$3 d$ & $4.498053096472\left(4.498055^{a}\right)$ & $0.0862527058258\left(0.086255^{a}\right)$ & $0.0451094116513\left(0.04511^{a}\right)$ & 0.0221116573287 \\
$5 d$ & 0.7042645932150 & 0.0240731854698 & 0.0177686031869 & 0.0105703836625 \\
$5 f$ & 0.6551355037045 & 0.0217548871225 & 0.0189834793491 & 0.0131097965268 \\
$5 g$ & 0.5870275279097 & 0.0203601943459 & 0.0197229049653 & 0.0161504601523 \\
\hline \hline
\end{tabular}

${ }^{a}$ Ref. [12].

TABLE V: Comparison of the calculated negative eigenvalues (in a.u.) with the literature for $C=1$ as functions of $B$. Literature results have been appropriately converted to the current scale of units.

\begin{tabular}{cllll}
\hline \hline State & $B=-25$ & $B=-10$ & $B=1$ & $B=10$ \\
\hline $1 s$ & $313.7035561801\left(313.7035^{a}\right)$ & $51.14471457780\left(51.14245^{a}\right)$ & 0.2562317633033 & 0.1170817257811 \\
$5 s$ & $0.9549949909076\left(0.9550^{a}\right)$ & $0.0619515214000\left(0.0615^{a}\right)$ & 0.0175035546929 & 0.0135892847495 \\
$5 d$ & $0.5235991105174\left(0.5236^{a}\right)$ & $0.0324039077933\left(0.0323^{a}\right)$ & 0.0198787392313 & 0.0192456899035 \\
$5 f$ & $0.1025542046267\left(0.10175^{a}\right)$ & $0.0200939350152\left(0.0201^{a}\right)$ & 0.0199927279413 & 0.0199358878025 \\
$5 g$ & $0.0200040154394\left(0.0200^{a}\right)$ & $0.0200014959681\left(0.0200^{a}\right)$ & 0.0199998554056 & 0.0199985879641 \\
\hline \hline
\end{tabular}

${ }^{a}$ Ref. [12].

states of $C=0.25$ were calculated for $B=-10,-1,1$ by [12] while for $C=1$, all the $n \leq 5$ states were reported for $B=-25,-10$ only, in the same work [12]. As in the previous tables, the present method offers noticeably improved results for all of these states compared to both of these. It appears that the variational calculations [12] are relatively more accurate in the low screening regions than in the stronger regions.

Now fig. 1 shows the variation of the radial probability distribution functions with respect to the interaction parameters $B$ and $C$; (a) shows this for $C=1$ at four $B$ values, viz., $-0.1,1,10$ and 100 while (b) shows this for $B=0.5$ at four $C$ values, viz., 0.01, 0.1, 1 and 10 respectively. It is seen that with an increase of $B$, the density distribution oozes out to the larger values of $r$ and the peak values get reduced, while exactly opposite behaviour is observed as the parameter $C$ is increased. Additionally, the radial density distributions of the Hellmann potential ( $B=1, C=10$ ) in fig. 2 shows the desired number nodes and peaks 

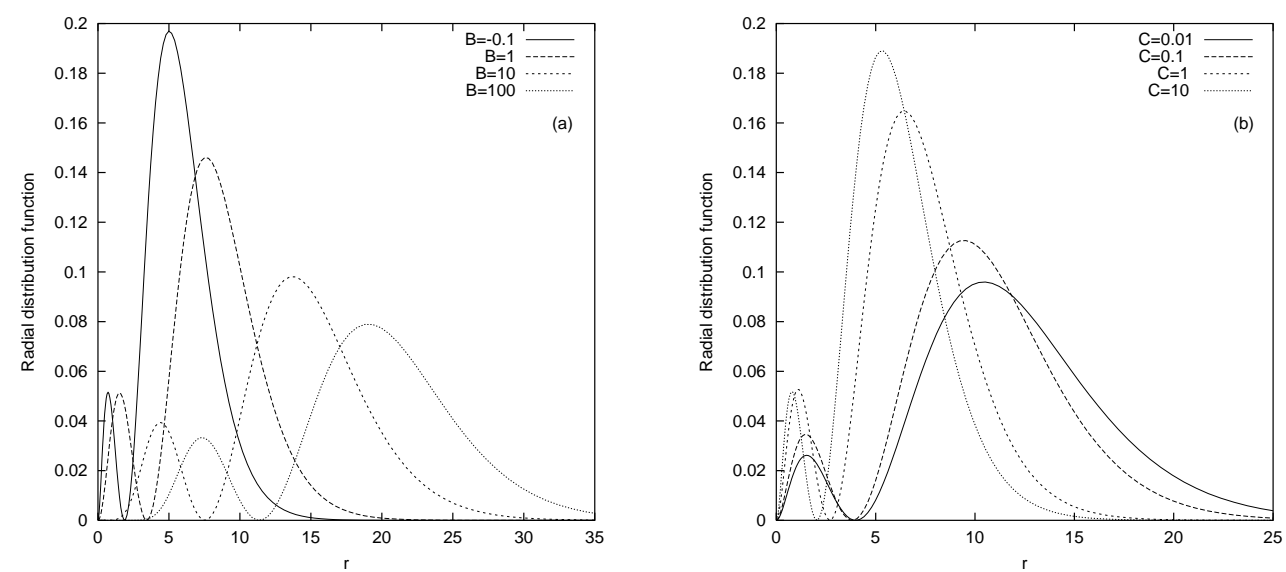

FIG. 1: Variation of the radial probability distribution function, $\left|r R_{n, \ell}\right|^{2}$, for $2 s$ state of the Hellmann potential with respect to the parameters $B$ and $C$. (a) $B=-0.1,1,10,100$ and $C=1$; (b) $B=0.5$ and $C=0.01,0.1,1,10$ respectively.

for the first three states in (b), (c) and (d) corresponding to $\ell=0,1$ respectively, along with the potential in (a).

Before passing, a few comments may be made. It is worth mentioning that although many attractive and elegant formalisms have been proposed over the years, it is usually quite a difficult task to achieve faster convergence and high accuracy results for the singular potentials at the same time by using the standard finite difference methods. As one author pointed out [24], a six- or seven-decimal place accuracy for the harmonic potential including an inverse quartic and sextic anharmonicity required at least 80,000 radial grid points for some of the lower states. The GPS method, employed in the current work possesses the simplicity of the finite difference and/or the finite element methods while simultaneously retaining the attractive features of the basis-set variational formalisms such as high accuracy and fast convergence. As already mentioned, all the calculations in this work have been done with only 200 radial grid points.

\section{CONCLUSION}

Discrete bound-state spectra of the superposed Coulomb and Yukawa potentials, are studied in detail by accurately calculating the eigenvalues and densities through the GPS method. The formalism is simple, computationally efficient, reliable and accurate. Low as well as high states are calculated for arbitrary values of the interaction parameters covering 

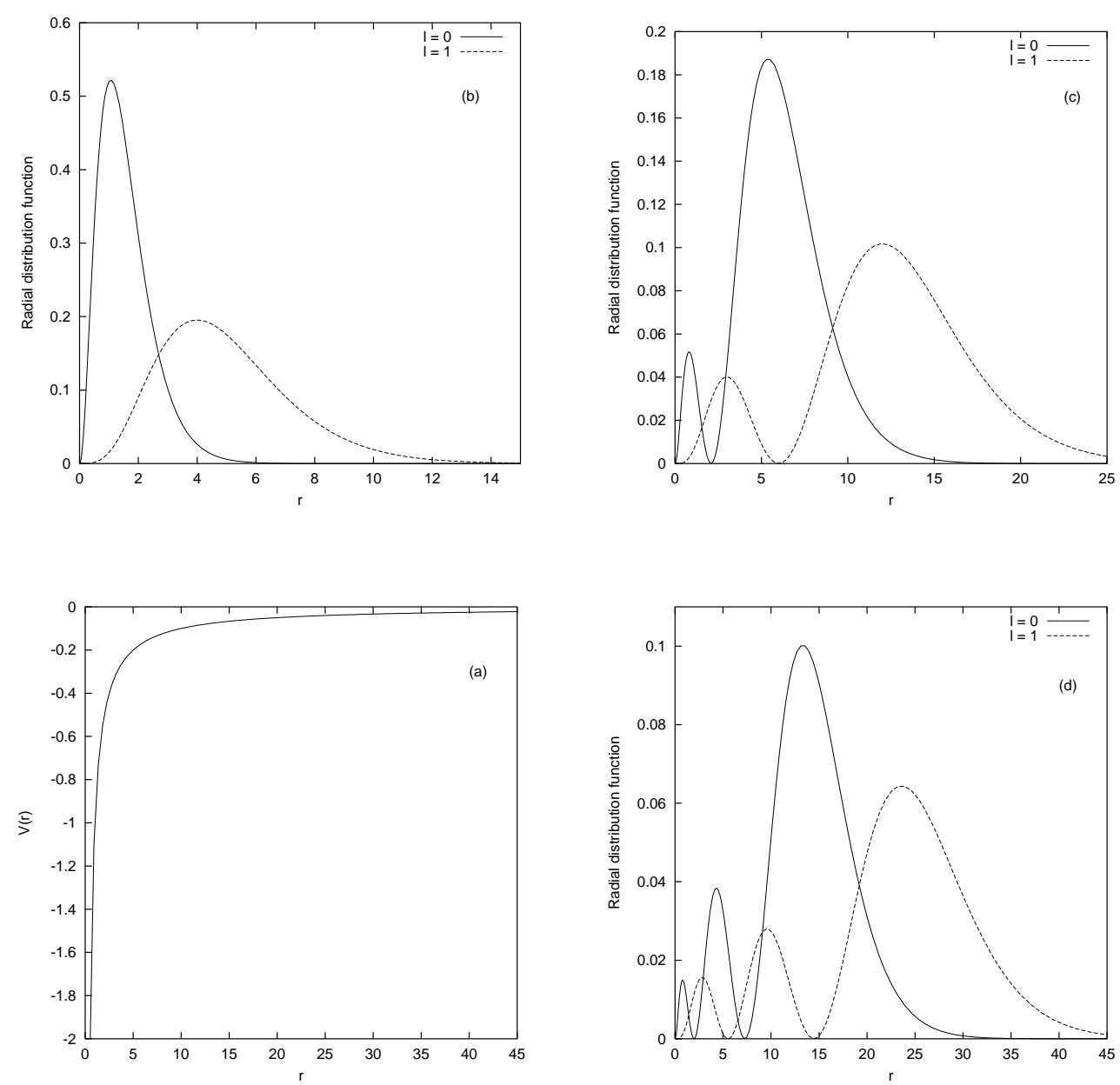

FIG. 2: Variation of the radial probability distribution functions, $\left|r R_{n, \ell}\right|^{2}$, for the first three states corresponding to $\ell=0,1$ of the Hellmann potential with $B=1$ and $C=10$. (a) the potential, (b) ground state, (c) first excited state and (d) second excited state.

weak and strong couplings with equal ease and accuracy. For all the 15 states belonging to $n \leq 5$, the present method offers results which significantly improves all other hitherto reported literature values. In view of the simplicity and accuracy offered by this method for the physical systems studied in this work, it might have equally successful and fruitful applications in various other branches of quantum mechanics.

\section{Acknowledgments}

AKR thanks Professors D. Neuhauser and S. I. Chu for useful discussions. He acknowledges the warm hospitality provided by the Univ. of California, Los angeles, CA, USA. EP 
gratefully acknowledges Q-Chem Inc., for support.

[1] H. Hellmann, Acta Physicochim, URSS 1, 913 (1935); ibid. 4, 225 (1936); ibid. 4, 324 (1936).

[2] H. Hellmann, J. Chem. Phys. 3, 61 (1935).

[3] H. Hellmann and W. Kassatotchkin, J. Chem. Phys. 4, 324 (1936).

[4] J. Callaway and P. S. Laghos, Phys. Rev. 187, 192 (1969).

[5] G. McGinn, J. Chem. Phys. 53, 3635 (1970).

[6] V. K. Gryaznov, M. V. Zhernokletov, V. N. Zubarev, I. L. Losilevskii and V. E. Tortov, Zh. Eksp. Teor. Fiz. 78, 573 (1980) [Sov. Phys. JETP. 51, 288 (1980)].

[7] V. A. Alekseev, V. E. Fortov and I. T. Yakubov, Usp. Fiz. Nauk. 139, (1983) [Sov. Phys. Usp. 26, 99 (1983)]

[8] J. N. Das and S. Chakravarty, Phys. Rev. A 32, 176 (1985).

[9] Y. P. Varshni and R. C. Shukla, Rev. Mod. Phys. 35, 130 (1963).

[10] J. C. Philips and L. Kleinmann, Phys. Rev. A 116, 287 (1959); ibid. 118, 1153 (1960).

[11] A. J. Hughes and J. Callaway, Phys. Rev. A 136, 1390 (1964).

[12] J. Adamowski, Phys. Rev. A 31, 43 (1985).

[13] R. Dutt, U. Mukherji and Y. P. Varshni, Phys. Rev. A 34, 777 (1986).

[14] M. Bag, R. Dutt and Y. P. Varshni, J. Phys. B 20, 5267 (1987).

[15] M. G. Kwato Njock, M. Nsangou, Z. Bona, S. G. Nana Engo and B. Oumarou, Phys. Rev. A 61, 042105 (2000).

[16] R. L. Hall and Q. D. Katabeh, Phys. Lett. A 287, 183 (2001).

[17] E. R. Vrscay, Phys. Rev. A 33, 1433 (1986).

[18] C. Stubbins, Phys. Rev. A 48, 220 (1993).

[19] M. A. Núñez, Phys. Rev. A 47, 3620 (1993). 1591 (2005).

[20] A. K. Roy, Phys. Lett. A 321, 231 (2004).

[21] A. K. Roy, J. Phys. G 30, 269 (2004).

[22] A. K. Roy, Int. J. Quant. Chem. 104, 861 (2005).

[23] A. K. Roy, Pramana-J. Phys. 65, 01 (2005).

[24] V. C. Aguilera-Navarro, G. A. Estévez and R. Guardiola, J. Math. Phys. 31, 99 (1990). 